\title{
Difference in Antibody Responses to Mycobacterium tuberculosis Antigens in Japanese Tuberculosis Patients Infected with the Beijing/Non-Beijing Genotype
}

\author{
Jingge Zhao, ${ }^{1}$ Beata Shiratori, ${ }^{2}$ Masao Okumura, ${ }^{3}$ Hideki Yanai, ${ }^{3}$ \\ Makoto Matsumoto, ${ }^{4}$ Chie Nakajima, ${ }^{5}$ Kazue Mizuno, ${ }^{3}$ Kenji Ono, ${ }^{4}$ Tetsuya Oda, \\ Haorile Chagan-Yasutan, ${ }^{2}$ Yugo Ashino, ${ }^{1}$ Takashi Matsuba, ${ }^{6}$ Takashi Yoshiyama, ${ }^{3}$ \\ Yasuhiko Suzuki, ${ }^{5}$ and Toshio Hattori, ${ }^{1,2,7}$ \\ ${ }^{1}$ Division of Emerging Infectious Diseases, Department of Internal Medicine, Graduate School of Medicine, Tohoku University, \\ Sendai, Miyagi 980-8574, Japan \\ ${ }^{2}$ Laboratory of Disaster-Related Infectious Disease, International Research Institute of Disaster Science, Tohoku University, \\ Sendai, Miyagi 980-8574, Japan \\ ${ }^{3}$ Fukujuji Hospital, Japan Anti-Tuberculosis Association, 3-1-2 4 Matsuyama, Kiyose, Tokyo 204-8533, Japan \\ ${ }^{4}$ Microbiological Research Institute, Otsuka Pharmaceutical Co., Ltd., Kawauchi-cho, Tokushima 771-0192, Japan \\ ${ }^{5}$ Division of Global Epidemiology, Research Center for Zoonosis Control, Hokkaido University, Sapporo, Hokkaido 001-0020, Japan \\ ${ }^{6}$ Division of Bacteriology, Department of Microbiology and Immunology, Faculty of Medicine, Tottori University, Yonago, \\ Tottori 683-8503, Japan \\ ${ }^{7}$ Graduate School of Health Science Studies, Kibi International University, 8 Igamachi, Takahashi 716-8508, Japan
}

Correspondence should be addressed to Toshio Hattori; hattorit@kiui.ac.jp

Received 18 October 2016; Accepted 8 December 2016; Published 15 January 2017

Academic Editor: Andréia M. Cardoso

Copyright (c) 2017 Jingge Zhao et al. This is an open access article distributed under the Creative Commons Attribution License, which permits unrestricted use, distribution, and reproduction in any medium, provided the original work is properly cited.

\begin{abstract}
The Beijing genotype Mycobacterium tuberculosis (MTB), notorious for its virulence and predisposition to relapse, could be identified by spoligotyping based on genetic heterogeneity. The plasma samples from 20 cases of Beijing and 16 cases of nonBeijing MTB infected individuals and 24 healthy controls (HCs) were collected, and antibodies against 11 antigens (Rv0679c142Asn, Rv0679c142Lys, Ag85B, Ag85A, ARC, TDM-M, TDM-K, HBHA, MDP-1, LAM, and TBGL) were measured by ELISA. Compared to the HCs, the MTB infected subjects showed higher titers of anti-Ag85B IgG (positivity 58.2\%) and anti-ACR IgG (positivity $48.2 \%$ ). Of note, anti-ACR IgG showed higher titer in Beijing MTB infected tuberculosis (TB) patients than in HC (Kruskal-Wallis test, $p<0.05$ ), while the levels of anti-Ag85B, anti-TBGL, anti-TDM-K, and anti-TDM-M IgG were higher in non-Beijing TB patients than in HC. Moreover, anti-Ag85B IgG showed higher response in non-Beijing TB patients than in Beijing TB patients $(p<0.05$; sensitivity, $76.9 \%$ versus $44.4 \%$ ). The sensitivity and specificity analysis showed that $78.8 \%$ Beijing infected individuals were negative in anti-TBGL-IgG or/and anti-Ag85B-IgG, while 75.0\% of those were positive in anti-TBGL-IgA or/and anti-ACR-IgG tests. These results indicate the possibility of developing antibody-based test to identify Beijing MTB.
\end{abstract}

\section{Introduction}

In 2013, tuberculosis (TB) infected 9 million individuals and caused 1.5 million deaths, making it one of the most critical infectious diseases worldwide [1]. Beijing genotype MTB has been most prevalent in East Asia [2], because of its virulence and resistance to chemical drugs and BCG vaccination [3].
Unfavorable treatment outcomes, including treatment failure and relapse, have also been found to be associated with the Beijing genotype MTB [4, 5]. Genotyping methods, such as spoligotyping and variable number tandem repeats typing (VNTR), have been commonly used to identify MTB genotypes, on the basis of polymerase chain reaction (PCR) $[6,7]$. However, the sensitivity of these tests pertains to 
acid-fast bacillus (AFB) smear results as low-AFB-positive samples $(\leq 1+)$ showed limited sensitivity of $<50 \%[8]$, and DNA-negative samples did not yield spoligopatterns [9]. This may be complemented by sputum culture, which may take 1-8 weeks for results at best [7]. Therefore, point-of-care testing (POCT) methods such as ELISA may help in quick differentiation of cases of Beijing and non-Beijing genotype MTB.

Rv0679c sequence is well conserved in MTB, M. bovis, and $M$. bovis BCG. The Rv0679c protein is a possible membrane lipoprotein located at the bacterial outer surface that may be involved in the entry of MTB into host cells $[10,11]$. Beijing genotype MTB carries a specific mutation on the $R v 0679 c$ gene, causing amino acid replacement at codon 142 from asparagine to lysine (Rv0679c142Lys) in contrast to the non-Beijing genotype MTB (Rv0679c142Asn) without this mutation [12]. Both TB Antigen 85A (Ag85A) and Antigen $85 \mathrm{~B}(\mathrm{Ag} 85 \mathrm{~B})$ are involved in mycobacterial cell wall assembly, possessing a mycolyl-transferase activity that plays a crucial role in the biogenesis of trehalose dimycolate (TDM) [13]. Ag85A may mainly facilitate MTB to survive in minimal nutritional medium [13], whereas Ag85B is reported as a potent immunoprotective antigen and a leading drug target $[14,15]$. Of note, Ag85B expression was reported to be lower in the Beijing/W lineage strains compared to the nonBeijing/W lineage strains [16, 17]. The $16-\mathrm{kDa} \alpha$-crystalline (ACR) protein of MTB, also known as HspX or Hsp 16.3, is required for MTB growth in macrophages [18] and is highly expressed in the presence of $\mathrm{NO}$ and low $\mathrm{O}_{2}$ concentration and in the stationary phase $[19,20]$, playing a role in the maintenance of long-term viability during latent, asymptomatic infections [18], which was found to be highly expressed in the Beijing group [16]. The heparin-binding hemagglutinin adhesin (HBHA) is indispensable for extrapulmonary dissemination [21], while the Beijing genotype MTB is more likely to develop extrapulmonary TB [22]. The surplus expression of mycobacterial DNA-binding protein 1 (MDP-1) in the slow-growing phase is a potential marker for latent TB infection (LTBI) for both non-Beijing and Beijing MTB [23]. TDM derivatives, including trehalose methoxydimycolate (TDM-M) and trehalose keto-dimycolate (TDM$\mathrm{K})$, from MTB $\mathrm{H} 37 \mathrm{Rv}$ constitute the major antigens of TBGL [24]. Anti-TBGL IgG and anti-lipoarabinomannan (LAM) antibodies were tested in the clinic as biomarkers for active TB infections (ATB) [25]. In our previous study, high plasma levels of anti-ACR, anti-LAM, anti-trehalose dimycolate (anti-TDM), and anti-TBGL IgG were found in patients with ATB, compared to those in the LTBI and control subjects [26]. Here we investigated the possibility of serological identification of Beijing/non-Beijing genotype MTB by using an in-house ELISA method.

\section{Materials and Methods}

2.1. Study Subjects. This study was approved by the Ethics Committee of Tohoku University and Fukujuji Hospital, number 2014-1-122. Signed informed consent was obtained from all study participants. All ATB subjects were diagnosed following the WHO guidelines on TB at Fukujuji
Hospital, Japan. For TB patient samples, only culture-positive samples were included. Blood samples were collected and stored before initiating anti-TB therapy, to avoid the effects of decreasing antibody titer during therapy [27]. Plasma samples were stored at $-30^{\circ} \mathrm{C}$ immediately after extraction without experiencing the repeated freeze-thaw cycle prior to the test. Twenty-six HCs were evaluated for LTBI by the TSPOT test (Oxford Immunotec, Inc., MA), in which 24 of them $(92.3 \%)$ were tested as negative; they were recruited in this study. In total, the plasma samples of $36 \mathrm{~TB}$ patients and $24 \mathrm{HCs}$ were tested under a double-blind study in which the results of spoligotyping test accomplished by Dr. Chie Nakajima and the results of ELISA test accomplished by Dr. Jingge Zhao were handed to the corresponding author Dr. Toshio Hattori who designed this study and who would perform the analysis.

2.2. MTB Diagnosis, Drug Susceptibility, Laboratory Test, and Chest Radiography. Acid-fast bacilli (AFB) smear staining and culture tests were performed to confirm MTB infection. Drug susceptibility test (DST) for isoniazid (INH), streptomycin (SM), rifampicin (RMP), and ethambutol (EMB) was conducted based on WHO-approved methods [28]. Laboratory data, including CRP, blood IgG, and IgA, were measured at Fukujuji Hospital. Chest radiographic data were read and summarized by three clinicians who had no access to other test results (Table 1).

2.3. Spoligotyping. The genotype of MTB clinical isolates was determined as described previously [29]. Briefly, the direct repeat (DR) region was amplified with a primer pair, and the PCR products were hybridized to a set of 43 spacer-specific oligonucleotide probes, which were covalently bound to the membrane. The spoligointernational type (SIT) was determined by comparing the spoligotypes against the international spoligotyping database (SpolDB4) [30].

2.4. Antigens. Gene cloning, expression, and purification method for Rv0679c was described previously [11]. Rv0679c142Asn and Rv0679c142Lys genes were cloned with the genomic DNA from non-Beijing and Beijing genotype MTB, respectively. The genes encoding Ag85A, Ag85B, ACR, HBHA, and MDP-1 were amplified by PCR from the genomic DNA of MTB H37Rv, and the primers were designed to incorporate restriction sites for cloning. The amplified DNA sequences were cloned into the pET-42 (+) vector (Novagen, South Africa), and the various vectors were expressed using Escherichia coli BL21 (DE3) (Novagen, South Africa). The recombinant proteins were purified via immobilized metal ion affinity chromatography using a nickel-sepharose high-performance column, according to standard protocols. The purity of the protein was analyzed by SDS-PAGE. The purified Lipoarabinomannan (LAM) (Nacalai Tesque, Kyoto, Japan) was isolated from MTB AoyamaB [31]. The purified trehalose methoxy-dimycolate (TDM-M) and trehalose ketodimycolate (TDM-K) were semisynthesized by chemically introducing methoxy-mycolic acid and keto-mycolic acid, respectively, to the $6,6^{\prime}$ position on trehalose. 
TABLE 1: Clinical characteristics.

\begin{tabular}{|c|c|c|c|}
\hline & $\begin{array}{c}\text { Non-Beijing } \\
n=16\end{array}$ & $\begin{array}{l}\text { Beijing } \\
n=20\end{array}$ & $p$ value \\
\hline \multicolumn{4}{|l|}{ Demographics } \\
\hline Males, $n(\%)$ & $14(87.5 \%)$ & $14(70.0 \%)$ & n.s. \\
\hline Age $[y$, mean $(\%)]$ & 50.7 & 60.2 & n.s. \\
\hline \multicolumn{4}{|l|}{ Mycobacterial identification } \\
\hline AFB-positive strains, $n(\%)$ & $13(81.2 \%)$ & $17(85.0 \%)$ & n.s. \\
\hline Time to growth in MGIT (weeks) & 7.43 & 8.20 & n.s. \\
\hline \multicolumn{4}{|l|}{ Drug resistance profile } \\
\hline Isoniazid-resistant strain & 0 & $3(15 \%)$ & n.s. \\
\hline MDR strain & $2(12.5 \%)$ & 0 & n.s. \\
\hline \multicolumn{4}{|l|}{ Laboratory findings } \\
\hline $\mathrm{CRP}(\mathrm{mg} / \mathrm{mL})$ & $6.55 \pm 4.38$ & $5.84 \pm 4.38$ & n.s. \\
\hline Blood IgG (mg/mL) & 17.67 & 16.12 & n.s. \\
\hline Blood IgA (mg/mL) & 3.83 & 3.99 & n.s. \\
\hline \multicolumn{4}{|l|}{ Chest radiograph findings } \\
\hline Cavity, positive (\%) & $4(26.7 \%)$ & $9(45.0 \%)$ & n.s. \\
\hline Pleural effusion, positive (\%) & $3(20.0 \%)$ & $5(25.0 \%)$ & n.s. \\
\hline
\end{tabular}

STD, standard deviation; MDR, multidrug resistance, as resistant to rifampin and isoniazid in this study; CRP, C-reactive protein.

2.5. ELISA. To detect the antibodies against dimorphic surface protein Rv0679c in MTB, Nunc MaxiSorp plates (Thermo Fisher Scientific, Inc., Waltham, MA) were coated with $100 \mu \mathrm{L}$ of $0.2 \mu \mathrm{g} / \mathrm{mL}$ purified recombinant Rv0679c142Asn or Rv0670c142Lys. Coated microplates were then allowed to stand overnight at $4^{\circ} \mathrm{C}$, which were then blocked with $3 \%$ $(\mathrm{w} / \mathrm{v})$ bovine serum albumin (BSA) in phosphate-buffered saline (PBS; $\mathrm{pH} 7.4$ ) for $4 \mathrm{~h}$. Plasma samples were diluted by 100 times in $1 \%(\mathrm{w} / \mathrm{v})$ BSA/PBS and then were added along with positive controls to set of triplicate wells. The positive controls were made of supernatant of mouse hybridoma cell culture that contained monoclonal antibody (mAb) 5D4-C2, which was diluted in 1/50-, 1/200-, and 1/800-fold dilution in $1 \% \mathrm{BSA} / \mathrm{PBS}$ [11]. The plates were sealed and incubated for $2 \mathrm{~h}$ at room temperature. After being washed 4 times with PBS- $0.05 \%$ Tween 20 (PBST), $100 \mu \mathrm{L}$ of $1: 10,000$ times diluted HRP-conjugated goat anti-human IgG heavy chain polyclonal antibody (LifeSpan BioSciences, Inc., Seattle, WA) and $100 \mu \mathrm{L}$ of $1: 10,000$ times diluted HRP-conjugated goat anti-mouse IgG antibody (Santa Cruz Biotechnology, Inc., Dallas, TX) in 1\% BSA/PBS were used as the second antibody for plasma samples and the mAb positive control, respectively. The sealed plates were then incubated for $2 \mathrm{~h}$ at room temperature. Then, the plates were washed 4 times and developed readable signal by $100 \mu \mathrm{L}$ of tetramethylbenzidine substrate (Dojindo, Kumamoto, Japan). To stop the reaction, $100 \mu \mathrm{L}$ of $0.5 \mathrm{M}$ sulfuric acid was added to each well, and the absorbance was read at $450 \mathrm{~nm}$. For other antigens, after $100 \mu \mathrm{L}$ of each of $4 \mu \mathrm{g} / \mathrm{mL}$ HBHA, $14 \mu \mathrm{g} / \mathrm{mL}$ Ag85A, $6 \mu \mathrm{g} / \mathrm{mL}$ Ag85B, $1 \mu \mathrm{g} / \mathrm{mL}$ ACR, $8 \mu \mathrm{g} / \mathrm{mL}$ MDP-1, $3 \mu \mathrm{g} / \mathrm{mL}$ TDM-M, $3 \mu \mathrm{g} / \mathrm{mL}$ TDM-K, and $0.5 \mu \mathrm{g} / \mathrm{mL}$ LAM were coated, similar methods were conducted to detect their specific corresponding IgG $[25,26]$. Plasma TBGL IgG and
TBGL IgA levels were measured using the Determiner TBGL Antibody ELISA Kit (Kyowa Medex Co., Ltd., Tokyo, Japan) [32].

2.6. Statistical Analysis. TB patients were grouped into Beijing and non-Beijing groups according to the results of spoligotyping. GraphPad Prism 6.0 (GraphPad Software, San Diego, CA) was used to analyze data and generate graphs. Comparison of clinical findings was achieved by nonparametric $t$-test and Chi-square test. Kruskal-Wallis test was used to evaluate differences in cases involving Beijing, non-Beijing, and HC groups. Dunn's post hoc tests were used to evaluate the differences between each pair following the Kruskal-Wallis test. Correlation analysis was accomplished by using Spearman correlation. The results were considered significant at $p<0.05$. The cutoff for anti-TBGL antibodies was set to 2 on the basis of previous studies [32]. The cutoffs for other antibodies were achieved by Youden's index by MedCalc (MedCalc Software bvba, Belgium).

\section{Results}

3.1. Genotypes of MTB as Identified by Spoligotyping. Spoligotyping data elucidated the phenotype of 36 clinical MTB strains, among which the Beijing genotype MTB accounted for 20 cases $(55.6 \%)$, while the non-Beijing genotype MTB accounted for 16 cases (44.4\%) (Table 1). Multiple drug resistance (MDR) MTB shows resistance to RMP and INH in T1 strain from non-Beijing MTB group. None of strains from Beijing/Non-Beijing group were resistant to SM and EMB. Although the non-Beijing genotype group consisted of 6 MTB subtypes, there was no significant difference in the response to each antigen in terms of subtypes (Table 2). 
TABLE 2: Non-Beijing genotype subgroups.

\begin{tabular}{lcccccc}
\hline & & \multicolumn{2}{c}{ Subtypes of non-Beijing MTB } & T2 & T3-OSA \\
Genotype & EAI2 Manila & LAM9 & New type & T1 & $n=5$ & $n=1$ \\
& $n=2$ & $n=1$ & $n=5$ & $2(40 \%)$ & 0 & $n=2$ \\
\hline Number of MDR strains (\%) & 0 & 0 & 0 & $1.37(0.33-2.29)$ & 0.310 & $1.89(1.76-2.03)$ \\
Anti-LAM IgG $^{\mathrm{a}}$ & $1.34(0.33-2.35)$ & 1.01 & $0.84(0.09-2.5)$ & $34.1(0.12-62.6)$ & 1.11 & $38.8(4.9-72.6)$ \\
Anti-TBGL IgG $^{\mathrm{a}}$ & $2.71(2.11-3.30)$ & 12.44 & $0.57(0.07-11.9)$ & $0.83(0.38-8.38)$ & 2.13 & $4.7(1.72-77.0)$ \\
Anti-TBGL IgA $^{\mathrm{a}}$ & $7.28(0.37-14.2)$ & 0.45 & $0.54(0.44-39.2)$ & $1.61(0.94-2.32)$ & 3.54 & 2.07 \\
Anti-Ag85B $^{\mathrm{a}}$ & $1.31(1.01-1.61)$ & 0.37 & $1.17(0.72-3.16)$ & $0.21(0.06-1.47)$ & 3.35 & 3.61 \\
Anti-ACR $^{\mathrm{a}}$ & $0.15(0.12-0.17)$ & 0.15 & $0.15(0.09-0.16)$ & 0.13 \\
\hline
\end{tabular}

${ }^{a}$ Median (range), EAI 2 Manila Clade EAI2 from the Manila family strain; LAM9, Latin American-Mediterranean; T1, specific T1 genotype clone (SIT number 266); T2, T2 Mycobacterium tuberculosis genotype.

3.2. Rv0679c ELISA. The ELISA test was optimized with regard to antigen concentration by using the monoclonal antibody 5D4-C2, which binds to both Asn142 and Lys142 without significant difference (Figure 1(a)). The ELISA assay for anti-Rv0679c142Asn IgG and anti-Rv0679c142Lys IgG showed that the majority in both non-Beijing and Beijing groups failed to react to each type of Rv0679c (Figure 1(b)), except for 2 samples in the non-Beijing group that showed higher reactivity to Rv0679c142Asn than Rv0679c142Lys and the 2 samples in the Beijing group that showed higher reactivity to Rv0679c142Lys than Rv0679c142Asn (Figure 1(c)).

3.3. IgG Antibodies against Ag85B, Ag85A, ACR, HBHA, $T D M-M$, TDM-K, MDP-1, and LAM. Of these 8 IgG antibodies, high titers of anti-Ag85 IgG were observed in the nonBeijing group compared to the Beijing group (Figure 2(a)). The comparison of IgG responses among non-Beijing, Beijing, and $\mathrm{HC}$ groups showed that the higher levels of antiAg85B IgG, anti-TDM-M IgG, and anti-TDM-K IgG were found in the non-Beijing group compared to the HC group (Figures 2(a), 2(e), and 2(f)). The anti-ACR IgG was higher in the Beijing group compared to the HC group (Figure 2(c)), while higher titers of anti-LAM IgG were found in both non-Beijing and Beijing groups compared to the HC group (Figure 2(h)). However, no significant difference was observed in the anti-Ag85A (Figure 2(b)), anti-HBHA (Figure 2(d)), and anti-MDP-1 (Figure 2(g)) IgG responses among the 3 groups.

3.4. Anti-TBGL Antibodies. The level of anti-TBGL IgG, rather than anti-TBGL IgA, was found to be higher in TB patients presenting with cavities (Figures 3(a) and 3(b)) as described elsewhere [32]. Despite the larger number of cavitypositive subjects in the Beijing group compared to the nonBeijing group ( $45.0 \%$ versus $26.7 \%$, nonsignificant, Table 1 ), no difference was noted in the anti-TBGL IgG response between the Beijing and non-Beijing groups. Notably, antiTBGL IgG showed higher titers in the non-Beijing and HC groups, but it did not differ in the Beijing group compared to the HC group (Figure 3(c)). On the other hand, anti-TBGL IgA was significantly higher in the Beijing group than in the HC group (Figure 3(d)). It is also noteworthy that all HCs were negative for anti-TBGL IgG.
3.5. Correlation Analysis. Significant correlations were observed between antibodies against lipid antigens and IgG against lipid-biosynthesis-related protein (Table 3). In detail, anti-Ag85B IgG titers correlated with antibodies against lipid $\mathrm{TB}$ antigens, including TDM-M/K, TBGL, and LAM. In contrast, anti-Ag85A IgG titers correlated with only antiTBGL IgA and with anti-TDM-M. Anti-LAM IgG were found to be correlating with anti-TDM-M/K IgG and anti-TBGL IgA/IgG.

3.6. Sensitivity and Specificity. Receiver operating characteristic (ROC) curve analysis was used to compare the sensitivity and specificity of each TB antibody test. The cutoff was determined based on all TB subjects and HCs. An antibody test that met the following conditions, specificity $>60 \%$ and sensitivity $>50 \%$ either in the non-Beijing or in the Beijing group, at $p<0.05$, was tentatively categorized as a prospective marker possessing discriminatory power, which includes anti-Ag85B IgG, anti-ACR IgG, anti-LAM IgG, and anti-TBGL IgG (Table 4). Of note, anti-Ag85B IgG showed higher response in the non-Beijing than in the Beijing group (76.9\% versus $44.4 \%$, Table 4 ), as opposed to anti-ACR IgG that showed higher response in the Beijing than in the nonBeijing group (61.1\% versus 30.8\%); both tests showed equal specificity up to $78.6 \%$. High specificity was observed for anti-TBGL IgG and anti-LAM IgG, up to $100 \%$ and $83.3 \%$, respectively. Combining anti-TBGL IgG and anti-LAM IgG increased the sensitivity up to $80 \%$ for the non-Beijing and $73.7 \%$ for the Beijing groups. Because anti-TBGL IgG were significantly higher in non-Beijing than in HC group, as well as higher anti-TBGL IgA in Beijing than in HC group, a test using the intersection of anti-TBGL IgG and anti-Ag85B IgG can yield higher accuracy to exclude the Beijing group within ATB (specificity, 77.8\%), while the union of anti-TBGL IgA and anti-ACR IgG can yield higher accuracy to detect the Beijing group within ATB (sensitivity, 75.0\%).

\section{Discussion}

In this study, high anti-ARC IgG and anti-TBGL IgA in Beijing TB infected patients, as well as high anti-Ag85B IgG and anti-TBGL IgG in Non-Beijing patients, could offer a sensitivity of $75.0 \%$ and a specificity of $77.8 \%$ for detecting Beijing MTB infection in diagnosed ATB. This can compensate the 


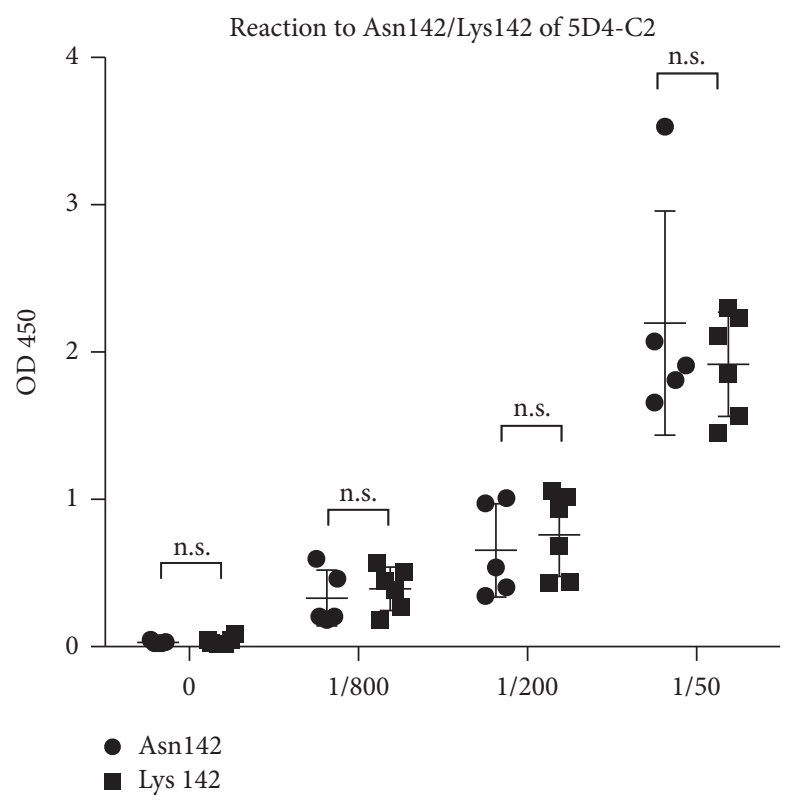

(a)

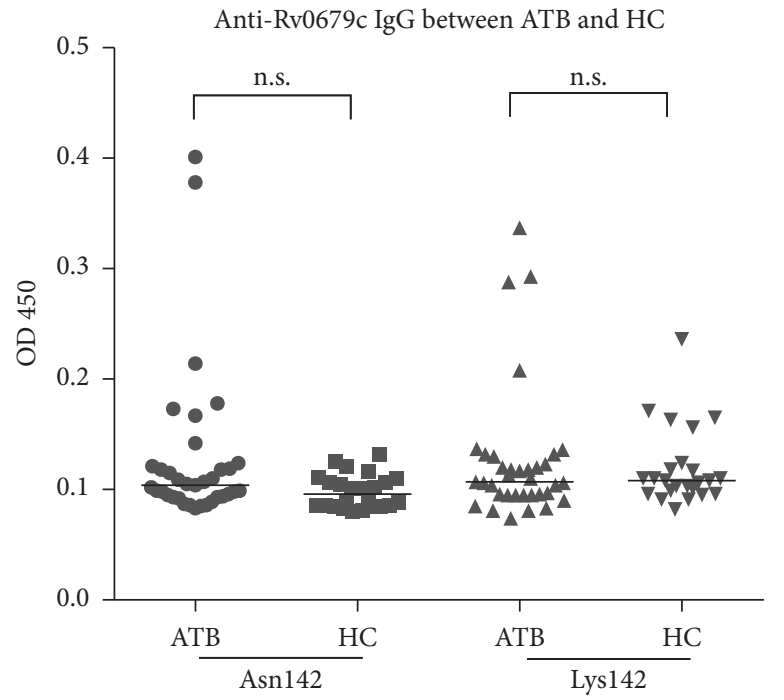

(b)

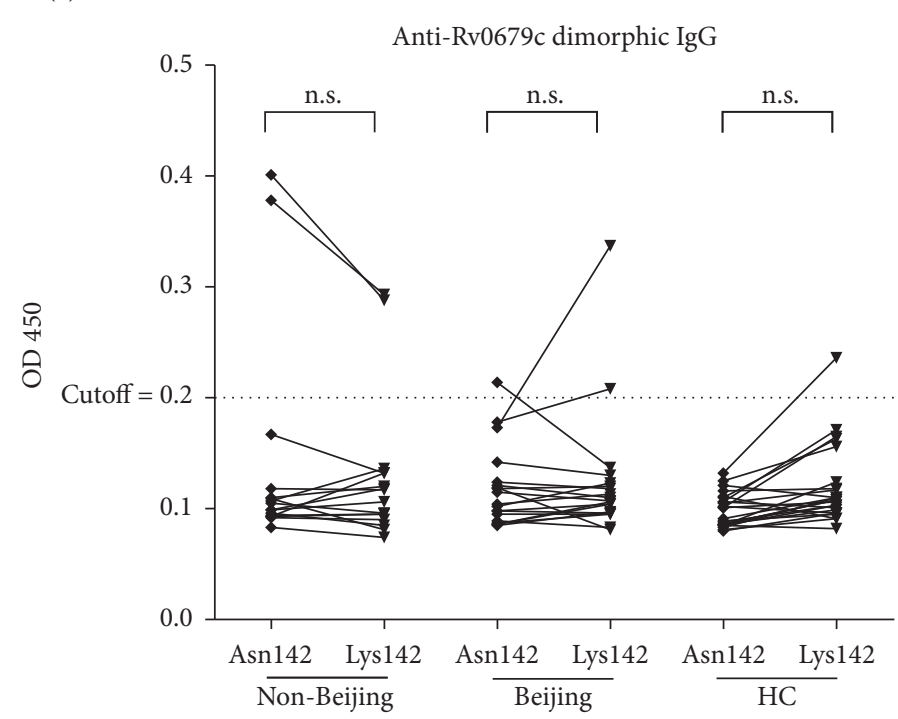

(c)

FIGURE 1: Antibody responses to Rv0679c142Asn and Rv0679c142Lys. (a) Anti-Rv0679c response observed at different dilutions of monoantibody 5D4C2 (0-, 1/50-, 1/200-, and 1/800-fold). (b) Anti-Asn142/Lys 142 IgG between the ATB and HC. Medians are indicated as lines. (c) Plasma antibody reaction to Rv0679c; 2 dots connected by a single line indicate a pair of reactions to Rv0679cAsn142 or Lys142 in one sample. The cutoff was set as twice the median of HC. n.s., not significant.

shortcoming of molecular diagnosis which needs bacteria derived DNA.

A mutated gene encoding the Lys142 variant of the Rv0679c protein has been found in the clinical isolates of the Beijing genotype family members [12]. The change caused by the Asn142Lys substitution may result in a reduced immune binding in response to a change of pathogenicity [33]. In this study, we observed a few specific recognitions of Rv0679c142Asn and Rv06790cLys142 antigens in the non-Beijing and Beijing groups, respectively, indicating discriminatory power upon IgG reactions against the distinct protein dimorphic between clades (Figure 1(c)). However, the reactions to MTB surface protein Rv0679c were not observed in the majority of samples (Figure 1(b)), agreeing with previous studies that found serological responses to MTB surface protein are relatively lower compared to those to cell wall component (LAM, e.g.) or secreted proteins [34]. Active TB infected (ATB) patients may lack high titerhigh avidity protective antibodies against the surface of MTB $[10,34]$.

Previous proteomics studies found that the expression of Ag85B appeared lower in Beijing genotype MTB in vitro $[16,17]$; therefore, lower titers of anti-Ag85B $\operatorname{IgG}$ in the Beijing group than in the non-Beijing group (Figure 2(a)) 


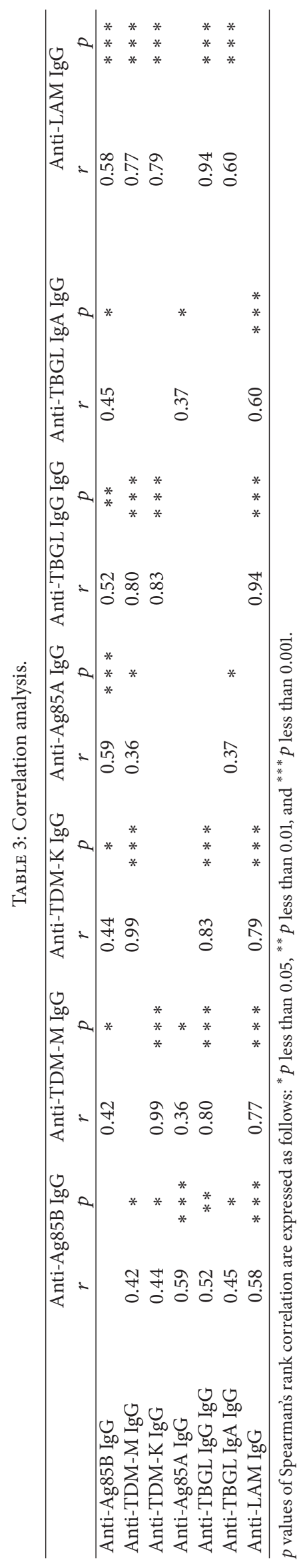




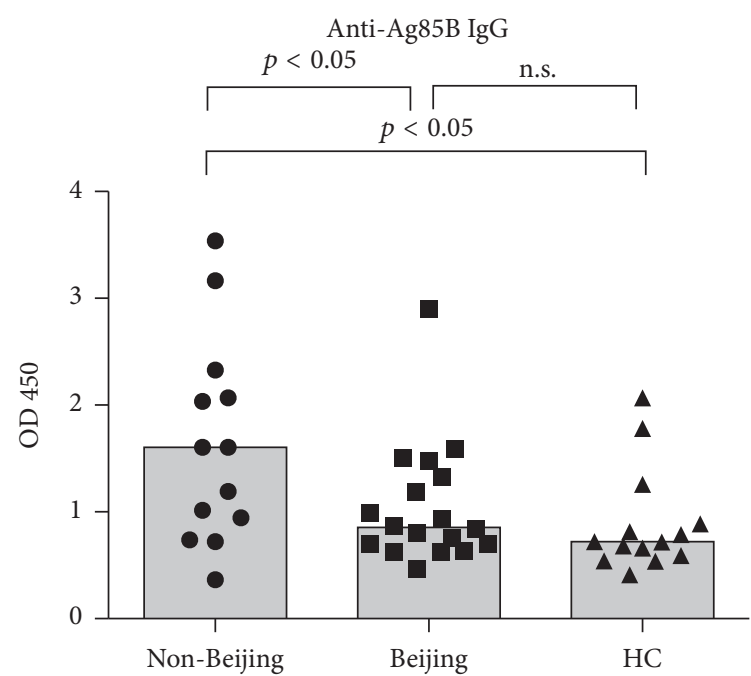

(a)

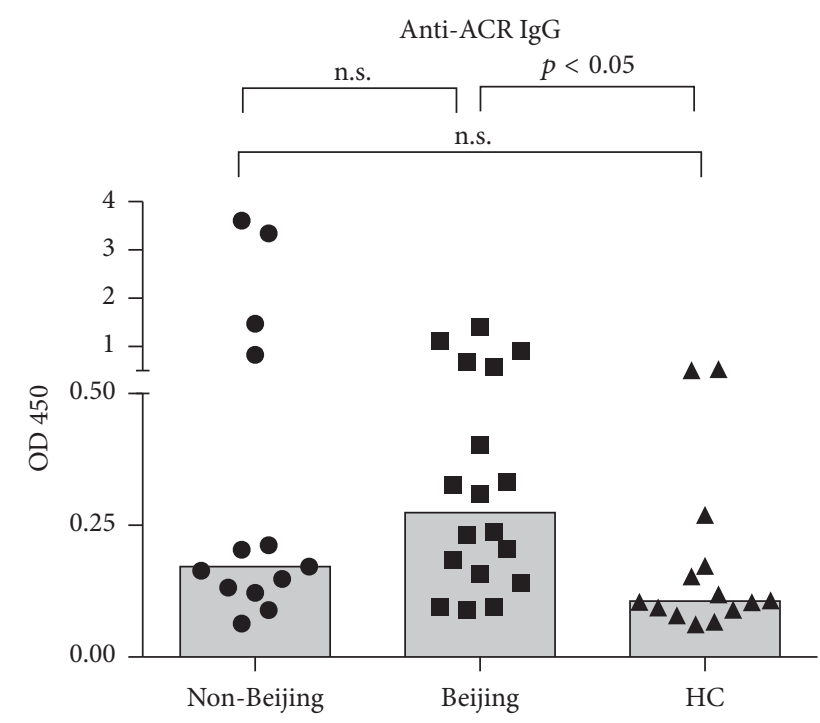

(c)

Anti-TDM-M IgG

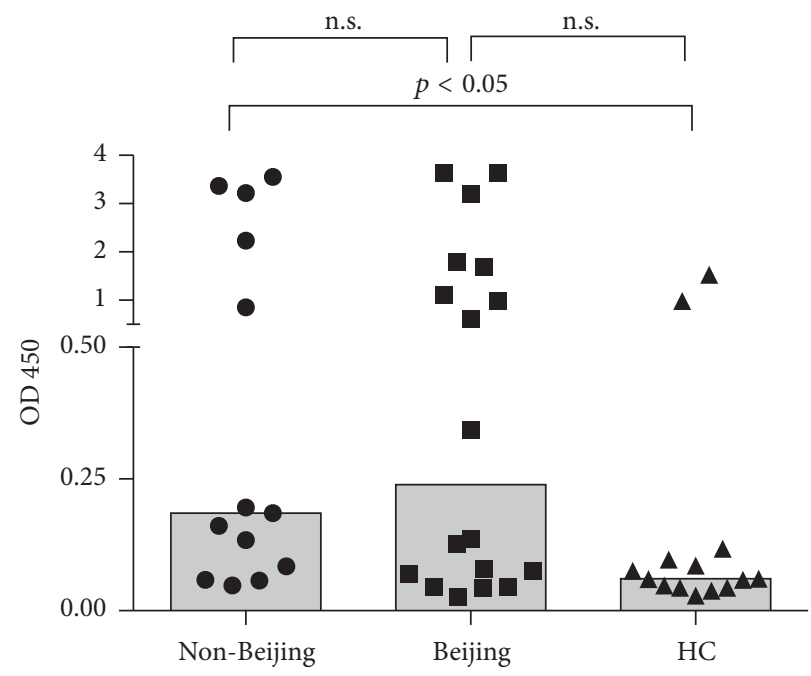

(e)

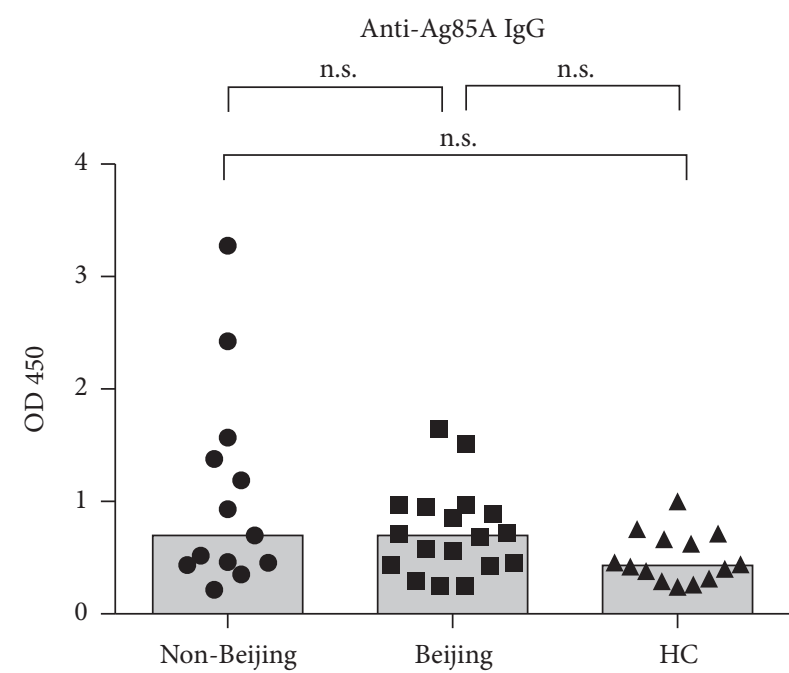

(b)

Anti-HBHA IgG

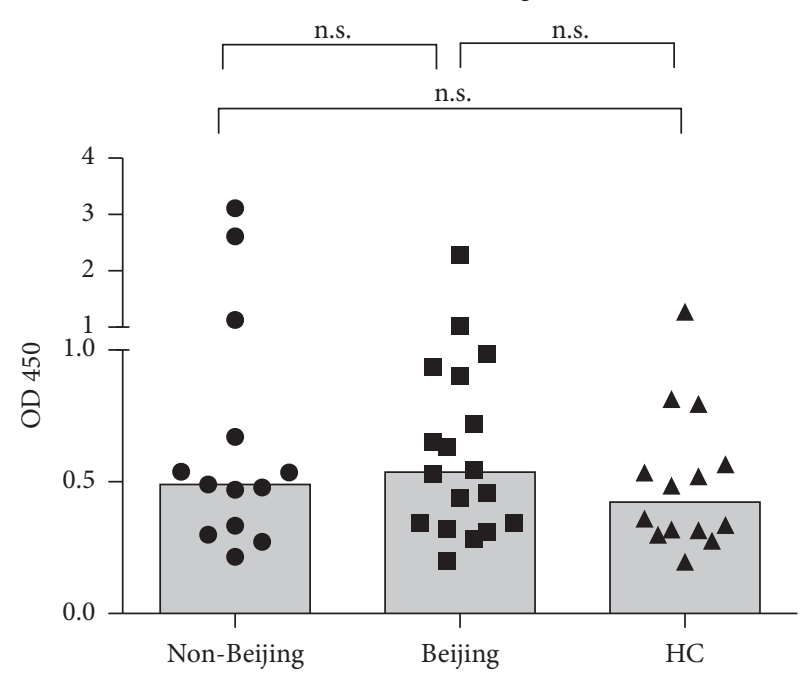

(d)

Anti-TDM-K IgG

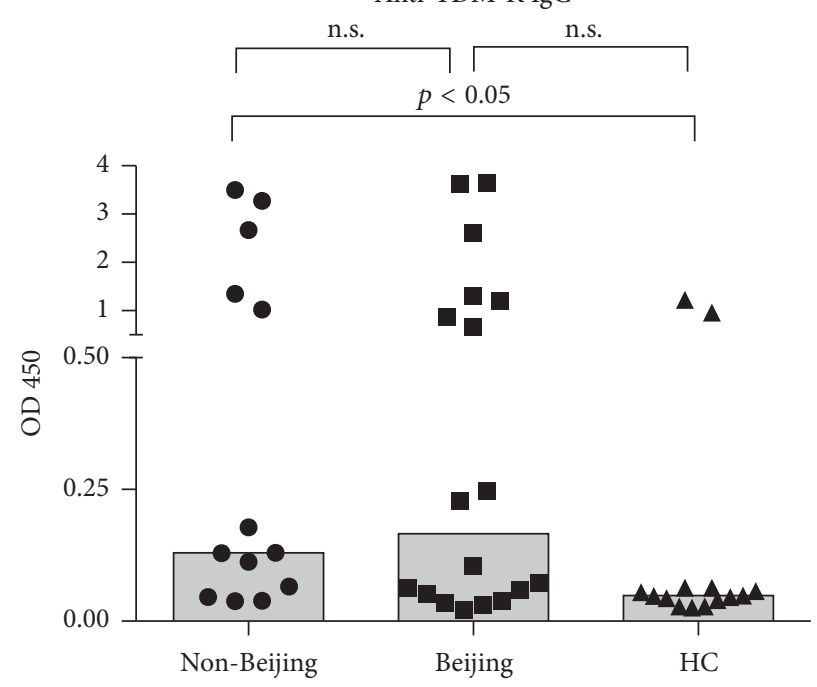

(f)

FIgure 2: Continued. 


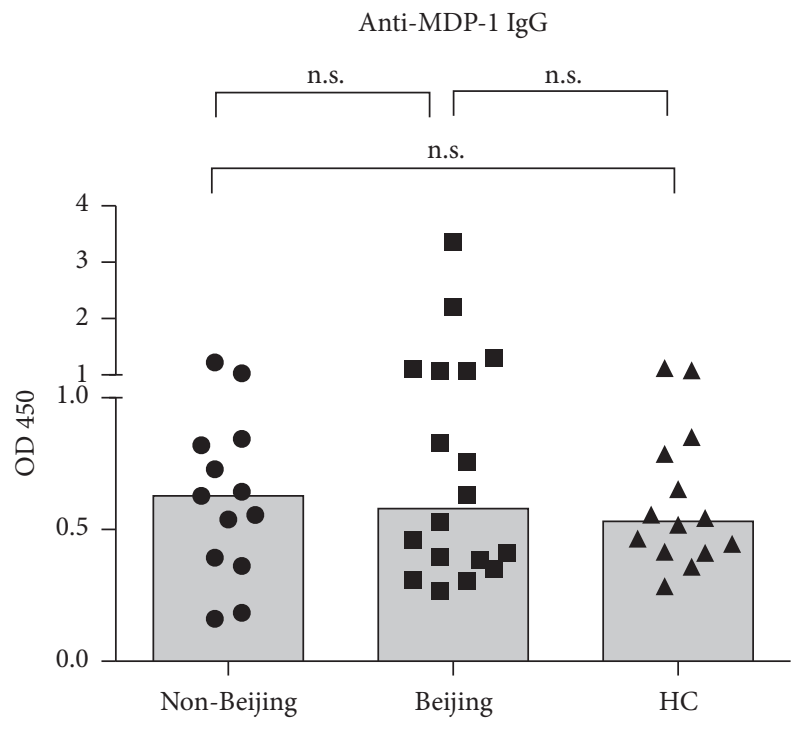

$(\mathrm{g})$

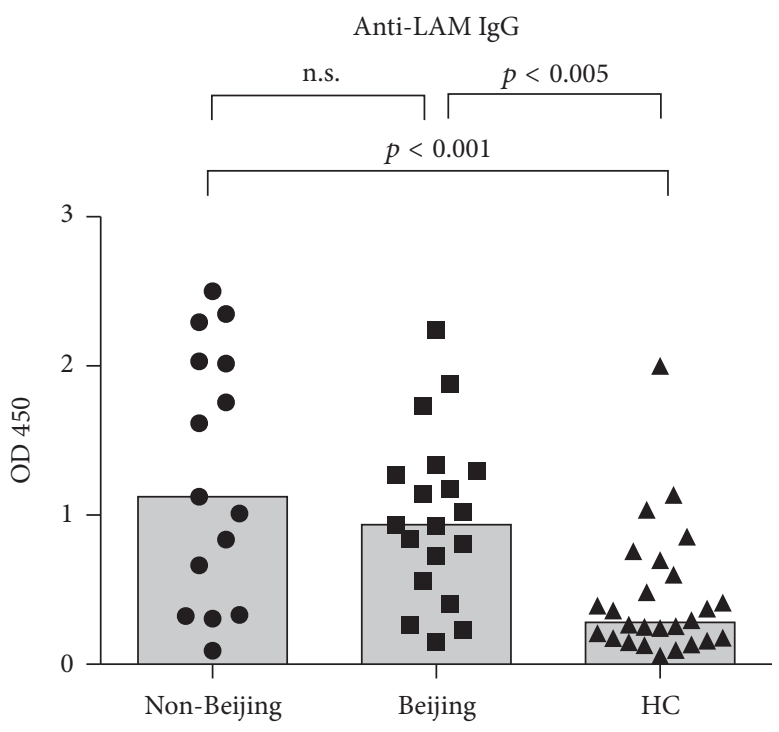

(h)

Figure 2: IgG antibody responses to Ag85B, Ag85A, ACR, HBHA, TDM-M, TDM-K, MDP-1, and LAM. (a) Anti-Ag85B IgG; (b) anti-Ag85A IgG; (c) anti-ACR IgG; (d) anti-HBHA IgG; (e) anti-TDM-M IgG; (f) anti-TDM-K IgG; (g) anti-MDP; (h) anti-LAM IgG responses. $p$ value $<0.05$ (Kruskal-Wallis test) indicated significant difference in the antibody response of the indicated groups. n.s., no significant. Medians are indicated as bars.

TABLE 4: Discriminatory power analysis.

\begin{tabular}{|c|c|c|c|c|c|c|}
\hline \multirow{2}{*}{ TB antibodies } & \multirow{2}{*}{ Cutoff $^{\mathrm{a}}$} & \multicolumn{3}{|c|}{ Sensitivity (\%) } & \multirow{2}{*}{$\begin{array}{c}\text { Specificity (\%) } \\
\text { HC }\end{array}$} & \multirow{2}{*}{$p$ value } \\
\hline & & TB subjects & Non-Beijing & Beijing & & \\
\hline Anti-Rv0679c142Asn IgG & 0.09 & 83.1 & 93.3 & 75.0 & 45.8 & 0.0301 \\
\hline Anti-Rv0679c142Lys IgG & 0.10 & 65.6 & 60.0 & 70.0 & 29.2 & n.s. \\
\hline Anti-Ag85B IgG & 0.89 & 58.2 & 76.9 & 44.4 & 78.6 & 0.035 \\
\hline Anti-Ag85A IgG & 0.76 & 42.0 & 46.2 & 38.9 & 92.9 & 0.0151 \\
\hline Anti-ACR IgG & 0.21 & 48.2 & 30.8 & 61.1 & 78.6 & 0.0024 \\
\hline Anti-HBHA IgG & 0.82 & 25.8 & 23.1 & 27.8 & 92.9 & n.s. \\
\hline Anti-TDM-M IgG & 0.50 & 41.9 & 38.5 & 44.4 & 85.7 & 0.0025 \\
\hline Anti-TDM-K IgG & 0.53 & 38.7 & 38.5 & 38.9 & 85.7 & 0.0031 \\
\hline Anti-MDPI IgG & 0.40 & 67.8 & 69.2 & 66.7 & 14.3 & n.s. \\
\hline Anti-LAM IgG & 0.76 & 67.7 & 66.7 & 68.4 & 83.3 & $<0.0001$ \\
\hline Anti-TBGL IgG (U/mL) & $2.00^{\mathrm{b}}$ & 55.6 & 62.5 & 50.0 & 100.0 & 0.0006 \\
\hline Anti-TBGL IgA (U/mL) & 1.00 & 42.9 & 40.0 & 45.0 & 80.1 & 0.0008 \\
\hline Anti-TBGL IgG (U/mL) or anti-LAM IgG & - & 76.5 & 80.0 & 73.7 & 83.3 & - \\
\hline Anti-TBGL IgG (U/mL) and anti-Ag85B IgG & - & 35.6 & 53.8 & 22.2 & 100.0 & - \\
\hline Anti-ACR IgG or anti-TBGL IgA (U/mL) & - & 59.4 & 40.0 & 75.0 & 64.3 & - \\
\hline
\end{tabular}

The sensitivity of the test to discriminate between non-Beijing and Beijing genotype TB was calculated in the context of the cutoff values obtained for all TB subjects. A $p$ value less than 0.05 indicates significant discriminatory power.

${ }^{a}$ Optimal cutoff was determined based on the ROC curve obtained for TB patients and healthy controls according to Youden's index.

${ }^{\mathrm{b}}$ Referring to the recommended cutoff, in accordance with the instructions of the anti-TBGL IgG kit.

may indicate lower burden of Ag85B during Beijing MTB infection [35]. Out of expectation, as Ag85B leads to the synthesis of TDM, we observed higher IgG against TDM$\mathrm{M}$, TDM-K, and TBGL in the non-Beijing group compared to the HC group (Figures 2 and 3), in addition to the significant correlation between anti-Ag85B IgG and antiTDMs IgG (anti-TDM-M IgG, $p<0.05$; anti-TDM-K and anti-TBGL IgG, $p<0.005$; Spearman correlation). For the first time, we reported higher tier of anti-Ag85B IgG in the non-Beijing group (OD median 1.605; positivity 76.9\%) compared those in the Beijing group (OD median 0.854; positivity $44.4 \%$ ). Since the stronger induction of anti-Ag85B IgG resulted in better protective effects in ATB [36], higher burden of Ag85B and the corresponding higher levels of antiAg85B IgG may confer to more protective immune responses during non-Beijing MTB infection rather than did Beijing 


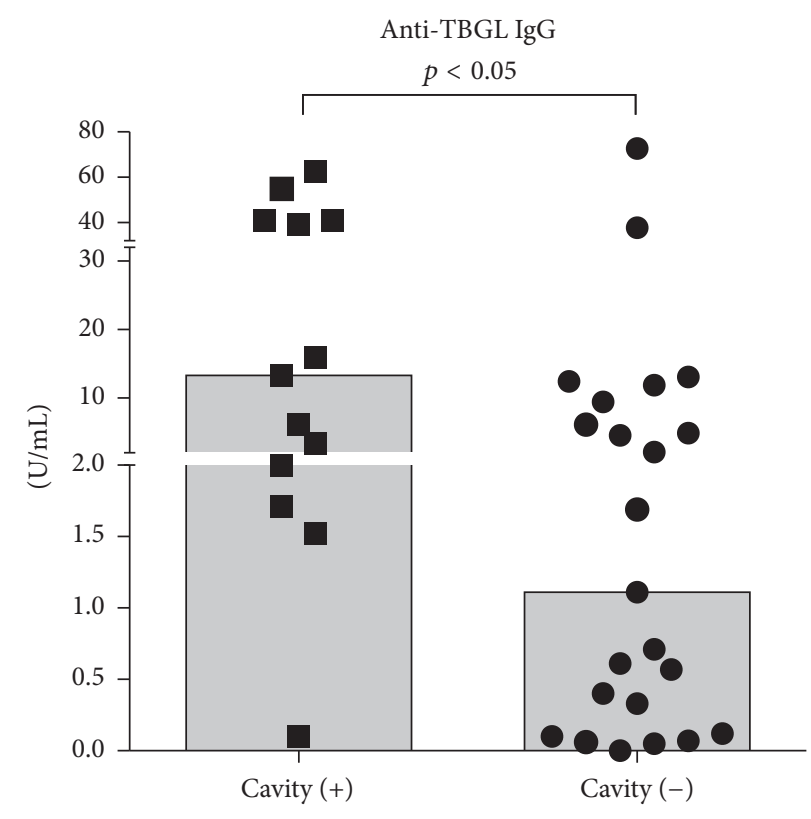

(a)

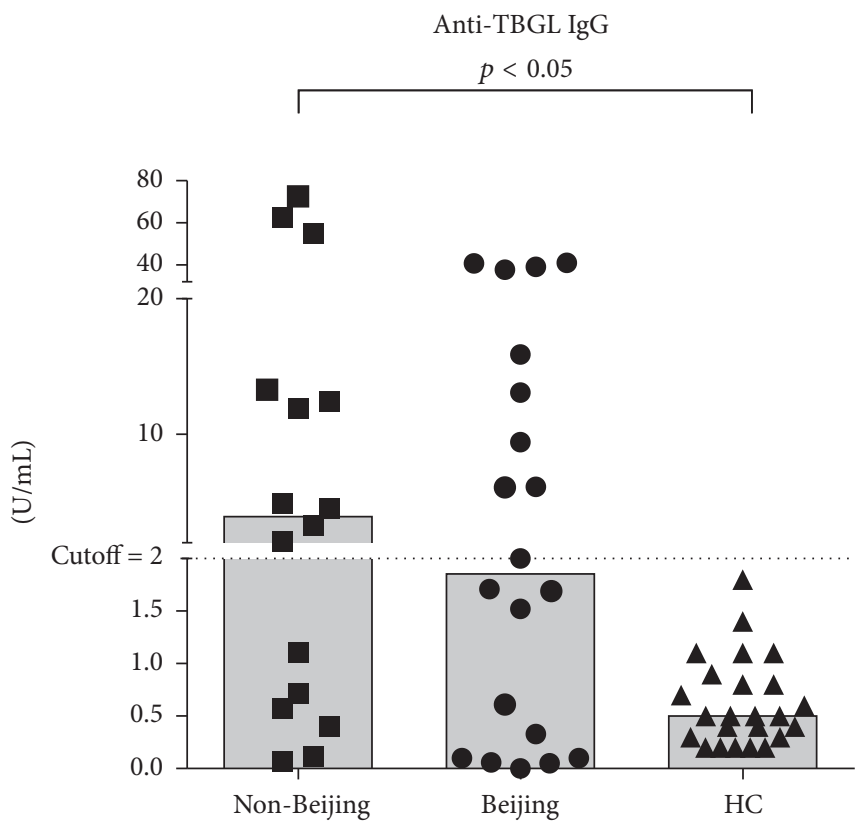

(c)

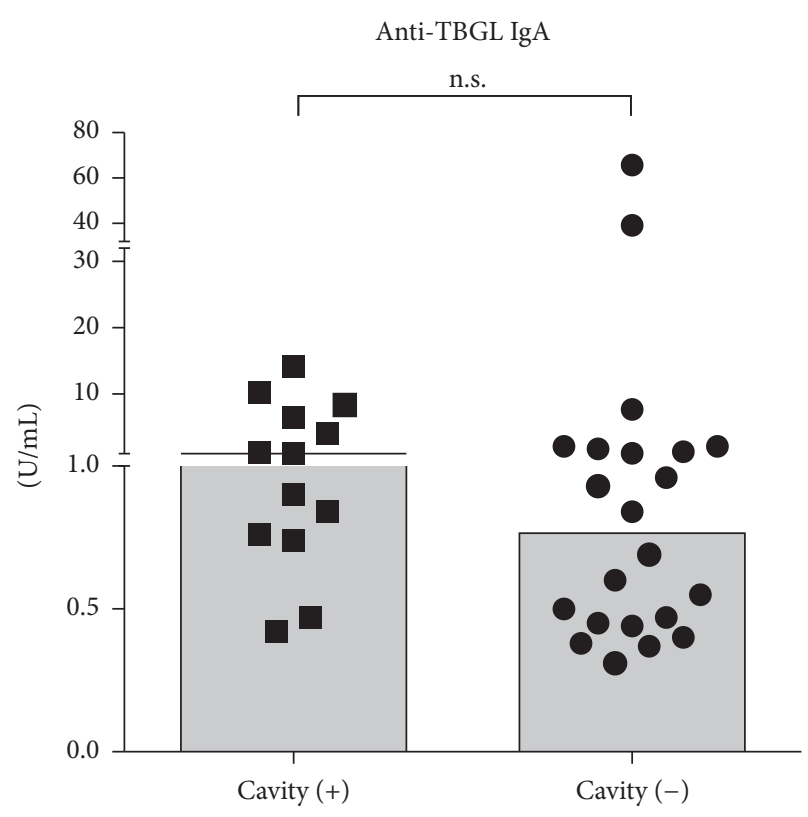

(b)

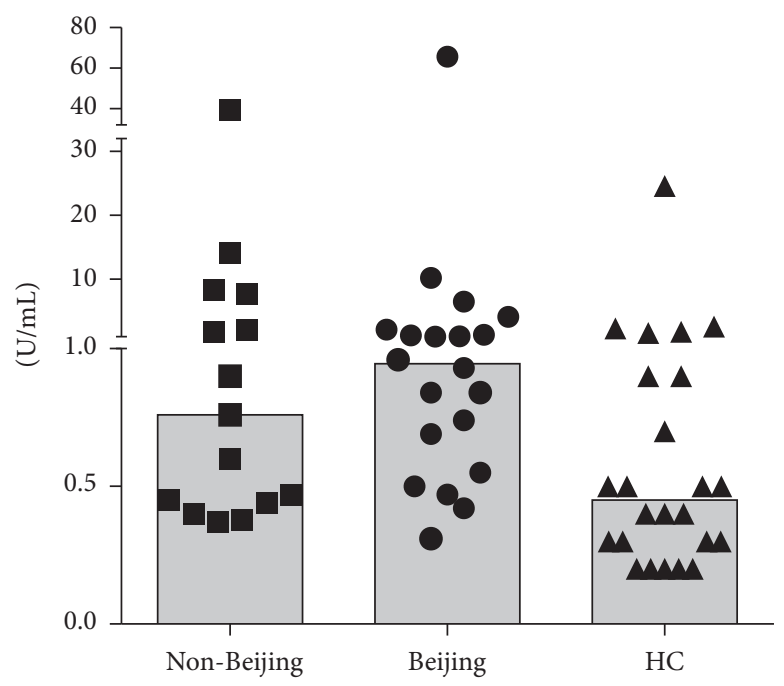

(d)

FIGURE 3: Association of anti-TBGL IgA and IgG responses and the presence of cavities and MTB genotype. $p$ value $<0.05$ indicated statistically significant difference. The cutoff of anti-TBGL IgG response is indicated in the instruction manual provided with the anti-TBGL IgG kit; (a), (b) cavities observed on chest radiographs; (c), (d) responses in non-Beijing and Beijing strain-infected individuals and HCs. Medians are indicated as bars.

MTB. Unlike Ag85B, Ag85A plays a role in support of the growth of MTB under nutritional stress, which is crucial for the persistence of MTB in macrophage cells. Lacking the antibody responses against Ag85A during active TB infection may be in association with the immune escape due to the fact of MTB intracellular infection [13], in contrast to elevated anti-Ag85A IgG that were found in individuals recovered from $\mathrm{TB}$ [37].
Higher IgG response against ACR was observed in the Beijing group compared to the HC group (Figure 2(c)), which is consistent with the finding of higher expression of ACR protein in the Beijing strains in vitro [16]. However, no significant difference was noted in the anti-ACR IgG titer between the Beijing and non-Beijing groups. Meanwhile, as the marker of extrapulmonary TB [38], anti-HBHA IgG were not found to be different among the three groups 
(Figure 2(d)), which can be explained by the risk factors for extrapulmonary TB infection that are related more to host than to MTB strain lineage characteristics [39].

Anti-LAM IgG responses were observed in patients with smear-positive, culture-positive sputum [40,41]. In spite of a significant correlation between anti-TBGL IgG and anti-LAM IgG, higher anti-TBGL IgG titers were observed in the nonBeijing group than in the HC group $(p<0.001)$. Meanwhile, significantly elevated anti-LAM IgG titers were observed in both the non-Beijing and Beijing group compared to the HC group, which was also reported in another study [35]. On the other hand, the anti-TBGL IgA titer in the Beijing group, instead of the non-Beijing group, appeared higher than those in the HC group. Of note, detecting anti-TBGL IgG is still practiced in TB diagnosis. TBGL mainly consists of TDM; therefore, the low anti-TBGL IgG levels in the Beijing genotype MTB group may be attributed to the small quantity of TDM that is associated with the low Ag85B expression in the Beijing group (Table 3). In our previous study in Indonesia where non-Beijing type MTB is prevalent up to $66.6 \%$ [42], $66.8 \%$ sensitivity of anti-TBGL IgG in detection of ATB was observed [26], which is higher than $55.6 \%$ sensitivity of that in this study where Beijing type TB infection made up a large proportion, suggesting anti-TBGL IgG titer be impacted by the infecting genotype of MTB. Moreover, in this study we found that all T-SPOT negative $\mathrm{HC}$ to be anti-TBGL IgG negative, while high anti-TBGL IgG were found in health care workers at the high risk of LTBI, indicating an association between anti-TBGL IgG and LTBI status [43]. In spite of the fact that limited samples are involved in this study, since anti-TBGL IgG is being used clinically irrespective of the MTB genotype the awareness is in need.

In a previous study, it has been observed that all the 7 individuals from the Haarlem MTB lineage-infected group showed positive reactions against TB Rv0407 antigen while only 2 out of 9 individuals from Latin-AmericanMediterranean MTB lineage-infected group showed such positive reaction [44]. Meanwhile, in another study, antiEsat6 IgG, anti-CFP10 IgG, and anti-LAM IgG responses were reported to be similar between Beijing and non-Beijing strain infection [35]. In conjunction with our result that anti-Ag85B IgG titers are higher in non-Beijing infection, these results suggest that the cornerstone for the genotype diagnosis method based on serologic responses should be built upon sophisticated screening for genotype-specific antigens. Therefore, a large number of TB antigens together with more plasma samples should be involved in future study.

\section{Conclusions}

In this study, we focused on serological antibody reactions against 11 antigens in terms of Beijing or non-Beijing genotype MTB infections by using a double-blind measure. IgG antibodies against Ag85B and TDM derivatives, including TDM-M, TDM-K, and TBGL, were found to be higher in non-Beijing genotype MTB infections compared to HC group, while anti-ACR IgG and anti-TBGL IgA were found to be higher in the Beijing compared to HC group. Despite the fact that out of 11 antigen-antibody tests only anti-Ag85B IgG showed higher response in non-Beijing TB patients than in Beijing TB patients $(p<0.05)$, the combination of antiAg85B IgG, anti-ACR IgG, and anti-TBGL antibodies tests could yield $75.0 \%$ sensitivity and $78.8 \%$ specificity for detecting Beijing MTB infection in ATB individual. Therefore, discrepancy in the antibody responses in the Beijing and nonBeijing groups could aid the identification of Beijing genotype MTB-specific infections.

\section{Competing Interests}

The authors declare that there is no conflict of interests regarding the publication of this paper.

\section{Acknowledgments}

This research is supported by the Research Program on Emerging and Re-Emerging Infectious Diseases from Japan Agency for Medical Research and Development, AMED. In addition, this study was also partially supported by a grant from the Ministry of Education, Culture, Sports, Science, and Technology of Japan for the Joint Research Program of the Research Center for Zoonosis Control at Hokkaido University and a special research grant from the International Research Institute of Disaster Science of Tohoku University. The authors would like to thank Dr. Katsue Ishi, Dr. Ken Fusano, Dr. Tadashi Idei, and Dr. Ai Ito from Fukujuji Hospital, Tokyo, Japan, who assisted in processing MTB strains and blood samples. All authors are grateful to Kyowa Medex Co. Ltd., Japan, for kindly providing Determiner TBGL Antibody ELISA Kit.

\section{References}

[1] WHO, Global Tuberculosis Report 2014, World Health Organization, Geneva, Switzerland, 2014.

[2] P. J. Bifani, B. Mathema, N. E. Kurepina, and B. N. Kreiswirth, "Global dissemination of the Mycobacterium tuberculosis WBeijing family strains," Trends in Microbiology, vol. 10, no. 1, pp. 45-52, 2002.

[3] I. Parwati, R. van Crevel, and D. van Soolingen, "Possible underlying mechanisms for successful emergence of the Mycobacterium tuberculosis Beijing genotype strains," The Lancet Infectious Diseases, vol. 10, no. 2, pp. 103-111, 2010.

[4] N. T. L. Hang, S. Maeda, N. Keicho, P. H. Thuong, and H. Endo, "Sublineages of Mycobacterium tuberculosis Beijing genotype strains and unfavorable outcomes of anti-tuberculosis treatment," Tuberculosis, vol. 95, no. 3, pp. 336-342, 2015.

[5] J. Wang, Y. Liu, C.-L. Zhang et al., "Genotypes and characteristics of clustering and drug susceptibility of mycobacterium tuberculosis isolates collected in heilongjiang province, China," Journal of Clinical Microbiology, vol. 49, no. 4, pp. 1354-1362, 2011.

[6] J. Bauer, Å. B. Andersen, K. Kremer, and H. Miörner, "Usefulness of spoligotyping to discriminate IS6110 low-copy-number Mycobacterium tuberculosis complex strains cultured in Denmark," Journal of Clinical Microbiology, vol. 37, no. 8, pp. 26022606, 1999. 
[7] B. Shiratori, S. Leano, C. Nakajima et al., "Elevated OPN, IP10 , and neutrophilia in loop-mediated isothermal amplification confirmed tuberculosis patients," Mediators of Inflammation, vol. 2014, Article ID 513263, 8 pages, 2014.

[8] P. I. Cafrune, L. G. Possuelo, A. W. Ribeiro et al., "Prospective study applying spoligotyping directly to DNA from sputum samples of patients suspected of having tuberculosis," Canadian Journal of Microbiology, vol. 55, no. 7, pp. 895-900, 2009.

[9] N. Suresh, J. Arora, H. Pant, T. Rana, and U. B. Singh, "Spoligotyping of Mycobacterium tuberculosis DNA from Archival Ziehl-Neelsen-stained sputum smears," Journal of Microbiological Methods, vol. 68, no. 2, pp. 291-295, 2007.

[10] D. P. Cifuentes, M. Ocampo, H. Curtidor et al., "Mycobacterium tuberculosis Rv0679c protein sequences involved in host-cell infection: potential TB vaccine candidate antigen," BMC Microbiology, vol. 10, article 109, 2010.

[11] T. Matsuba, Y. Suzuki, and Y. Tanaka, "Association of the Rv0679c protein with lipids and carbohydrates in Mycobacterium tuberculosis/Mycobacterium bovis BCG," Archives of Microbiology, vol. 187, no. 4, pp. 297-311, 2007.

[12] C. Nakajima, A. Tamaru, Z. Rahim et al., "Simple multiplex PCR assay for identification of Beijing family Mycobacterium tuberculosis isolates with a lineage-specific mutation in Rv0679c," Journal of Clinical Microbiology, vol. 51, no. 7, pp. 2025-2032, 2013.

[13] L. Y. Armitige, C. Jagannath, A. R. Wanger, and S. J. Norris, "Disruption of the genes encoding antigen 85A and antigen 85B of Mycobacterium tuberculosis H37Rv: effect on growth in culture and in macrophages," Infection and Immunity, vol. 68, no. 2, pp. 767-778, 2000.

[14] H. G. Wiker and M. Harboe, "The antigen 85 complex: a major secretion product of Mycobacterium tuberculosis," Microbiological Reviews, vol. 56, no. 4, pp. 648-661, 1992.

[15] A. Geluk, K. E. Van Meijgaarden, K. L. M. C. Franken et al., "Identification of major epitopes of Mycobacterium tuberculosis AG85B that are recognized by HLA-A ${ }^{*} 0201$-restricted $\mathrm{CD} 8^{+} \mathrm{T}$ cells in HLA-transgenic mice and humans," Journal of Immunology, vol. 165, no. 11, pp. 6463-6471, 2000.

[16] F. Li, H. Li, W.-Z. Zuo et al., "The viability and protein expression of Beijing/W Lineage mycobacterium tuberculosis circulating in Xinjiang, China," Current Microbiology, vol. 70, no. 5, pp. 735-744, 2015.

[17] C. Pheiffer, J. C. Betts, H. R. Flynn, P. T. Lukey, and P. van Helden, "Protein expression by a Beijing strain differs from that of another clinical isolate and Mycobacterium tuberculosis H37Rv," Microbiology, vol. 151, no. 4, pp. 1139-1150, 2005.

[18] Y. Yuan, D. D. Crane, R. M. Simpson et al., "The $16-\mathrm{kDa}$ $\alpha$-crystallin (Acr) protein of Mycobacterium tuberculosis is required for growth in macrophages," Proceedings of the National Academy of Sciences of the United States of America, vol. 95, no. 16, pp. 9578-9583, 1998.

[19] A. Purkayastha, L. A. McCue, and K. A. McDonough, "Identification of a Mycobacterium tuberculosis putative classical nitroreductase gene whose expression is coregulated with that of the acr gene within macrophages, in standing versus shaking cultures, and under low oxygen conditions," Infection and Immunity, vol. 70, no. 3, pp. 1518-1529, 2002.

[20] Y. Yuan, D. D. Crane, and C. E. Barry III, "Stationary phaseassociated protein expression in Mycobacterium tuberculosis: function of the mycobacterial $\alpha$-crystallin homolog," Journal of Bacteriology, vol. 178, no. 15, pp. 4484-4492, 1996.
[21] K. Pethe, S. Alonso, F. Biet et al., "The heparin-binding haemagglutinin of $\mathrm{M}$. tuberculosis is required for extrapulmonary dissemination," Nature, vol. 412, no. 6843, pp. 190-194, 2001.

[22] Y. Kong, M. D. Cave, L. Zhang et al., "Association between Mycobacterium tuberculosis Beijing/W lineage strain infection and extrathoracic tuberculosis: insights from epidemiologic and clinical characterization of the three principal genetic groups of M. tuberculosis clinical isolates," Journal of Clinical Microbiology, vol. 45, no. 2, pp. 409-414, 2007.

[23] S. Matsumoto, M. Furugen, H. Yukitake, and T. Yamada, “The gene encoding mycobacterial DNA-binding protein I (MDPI) transformed rapidly growing bacteria to slowly growing bacteria," FEMS Microbiology Letters, vol. 182, no. 2, pp. 297-301, 2000.

[24] Y. Fujita, T. Naka, M. R. McNeil, and I. Yano, "Intact molecular characterization of cord factor (trehalose 6,6I-dimycolate) from nine species of mycobacteria by MALDI-TOF mass spectrometry," Microbiology, vol. 151, no. 10, pp. 3403-3416, 2005.

[25] J. Zhao, Z. Zhu, X. Zhang et al., "Evaluation of anti-TBGL antibody in the diagnosis of tuberculosis patients in China," Journal of Immunology Research, vol. 2015, Article ID 834749, 9 pages, 2015.

[26] M. A. Senoputra, B. Shiratori, F. M. Hasibuan et al., "Diagnostic value of antibody responses to multiple antigens from Mycobacterium tuberculosis in active and latent tuberculosis," Diagnostic Microbiology and Infectious Disease, vol. 83, no. 3, pp. 278-285, 2015.

[27] A. Azzurri, G. V. Kanaujia, O. Y. Sow et al., "Serological markers of pulmonary tuberculosis and of response to anti-tuberculosis treatment in a patient population in Guinea," International Journal of Immunopathology and Pharmacology, vol. 19, no. 1, pp. 199-208, 2006.

[28] WHO, Drug Susceptibility Testing, World Health Organization, Geneva, Switzerland, 2012.

[29] J. Kamerbeek, L. Schouls, A. Kolk et al., "Simultaneous detection and strain differentiation of Mycobacterium tuberculosis for diagnosis and epidemiology," Journal of Clinical Microbiology, vol. 35, no. 4, pp. 907-914, 1997.

[30] K. Brudey, J. R. Driscoll, L. Rigouts et al., "Mycobacterium tuberculosis complex genetic diversity: mining the fourth international spoligotyping database (SpolDB4) for classification, population genetics and epidemiology," BMC Microbiology, vol. 6, article no. 23, 2006.

[31] B. Hamasur, G. Källenius, and S. B. Svenson, "A new rapid and simple method for large-scale purification of mycobacterial lipoarabinomannan," FEMS Immunology and Medical Microbiology, vol. 24, no. 1, pp. 11-17, 1999.

[32] M. Mizusawa, M. Kawamura, M. Takamori et al., "Increased synthesis of anti-tuberculous glycolipid immunoglobulin G (IgG) and IgA with cavity formation in patients with pulmonary tuberculosis," Clinical and Vaccine Immunology, vol. 15, no. 3, pp. 544-548, 2008.

[33] T. Matsuba, U. R. Siddiqi, T. Hattori et al., "Antigenic characterization of dimorphic surface protein in Mycobacterium tuberculosis," FEMS Microbiology Letters, vol. 363, no. 10, 2016.

[34] C. C. Perley, M. Frahm, E. M. Click et al., "The human antibody response to the surface of Mycobacterium tuberculosis," PLoS ONE, vol. 9, no. 6, Article ID e98938, 2014.

[35] Y.-G. Hur, A. Kim, Y. A. Kang et al., "Evaluation of antigenspecific immunoglobulin g responses in pulmonary tuberculosis patients and contacts," Journal of Clinical Microbiology, vol. 53, no. 3, pp. 904-909, 2015. 
[36] F. Abebe and G. Bjune, "The protective role of antibody responses during Mycobacterium tuberculosis infection," Clinical and Experimental Immunology, vol. 157, no. 2, pp. 235-243, 2009.

[37] M. Osada-Oka, Y. Tateishi, Y. Hirayama et al., "Antigen 85A and mycobacterial DNA-binding protein 1 are targets of immunoglobulin G in individuals with past tuberculosis," Microbiology and Immunology, vol. 57, no. 1, pp. 30-37, 2013.

[38] A.-R. Shin, K.-S. Lee, J.-S. Lee et al., "Mycobacterium tuberculosis HBHA protein reacts strongly with the serum immunoglobulin M of tuberculosis patients," Clinical and Vaccine Immunology, vol. 13, no. 8, pp. 869-875, 2006.

[39] T. Gomes, S. A. Vinhas, B. Reis-Santos et al., "Extrapulmonary tuberculosis: Mycobacterium tuberculosis strains and host risk factors in a large urban setting in Brazil," PLoS ONE, vol. 8, no. 10, Article ID e74517, 2013.

[40] T. A. Tessema, G. Bjune, B. Hamasur, S. Svenson, H. Syre, and B. Bjorvatn, "Circulating antibodies to lipoarabinomannan in relation to sputum microscopy, clinical features and urinary anti-lipoarabinomannan detection in pulmonary tuberculosis," Scandinavian Journal of Infectious Diseases, vol. 34, no. 2, pp. 97-103, 2002.

[41] X. Yu, R. Prados-Rosales, E. R. Jenny-Avital, K. Sosa, A. Casadevall, and J. M. Achkar, "Comparative evaluation of profiles of antibodies to mycobacterial capsular polysaccharides in tuberculosis patients and controls stratified by HIV status," Clinical and Vaccine Immunology, vol. 19, no. 2, pp. 198-208, 2012.

[42] I. Parwati, B. Alisjahbana, L. Apriani et al., "Mycobacterium tuberculosis beijing genotype is an independent risk factor for tuberculosis treatment failure in Indonesia," Journal of Infectious Diseases, vol. 201, no. 4, pp. 553-557, 2010.

[43] U. R. Siddiqi, P. S. A. Leano, H. Chagan-Yasutan et al., "Frequent detection of anti-tubercular-glycolipid-IgG and -IgA antibodies in healthcare workers with latent tuberculosis infection in the Philippines," Clinical and Developmental Immunology, vol. 2012, Article ID 610707, 10 pages, 2012.

[44] P. Schierloh, L. Klepp, C. Vazquez et al., "Differential expression of immunogenic proteins on virulent Mycobacterium tuberculosis clinical isolates," BioMed Research International, vol. 2014, Article ID 741309, 13 pages, 2014. 


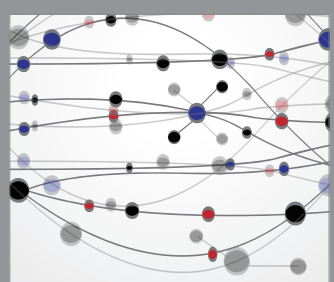

The Scientific World Journal
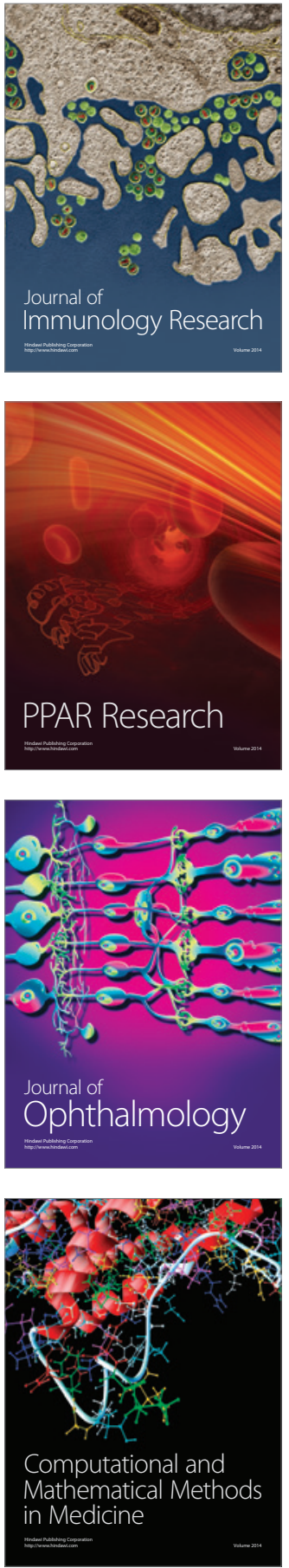

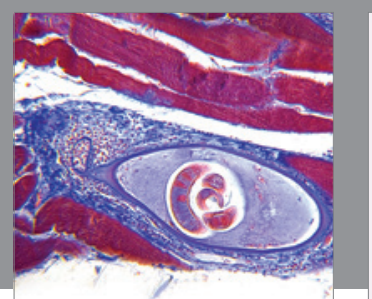

Gastroenterology Research and Practice
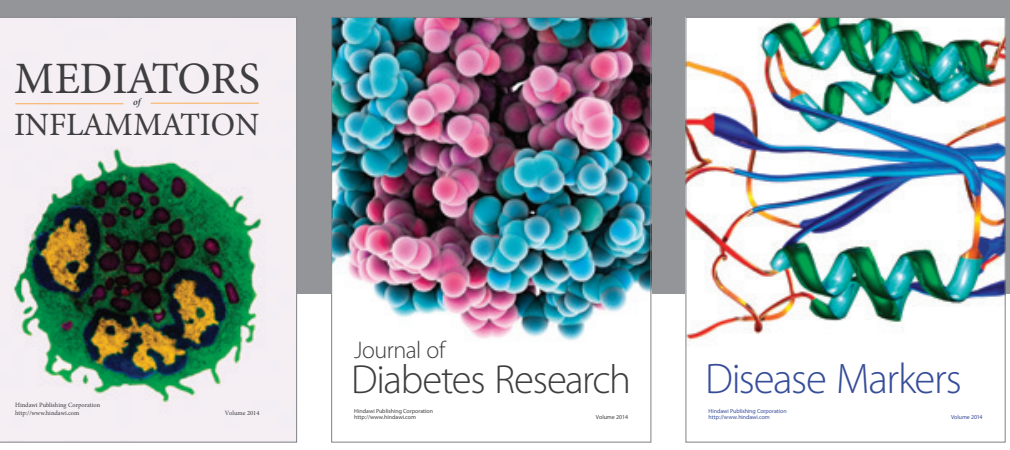

Disease Markers

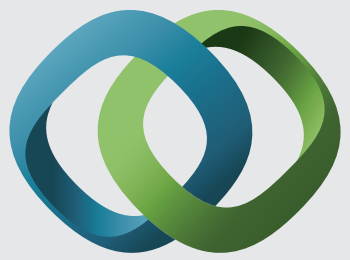

\section{Hindawi}

Submit your manuscripts at

https://www.hindawi.com
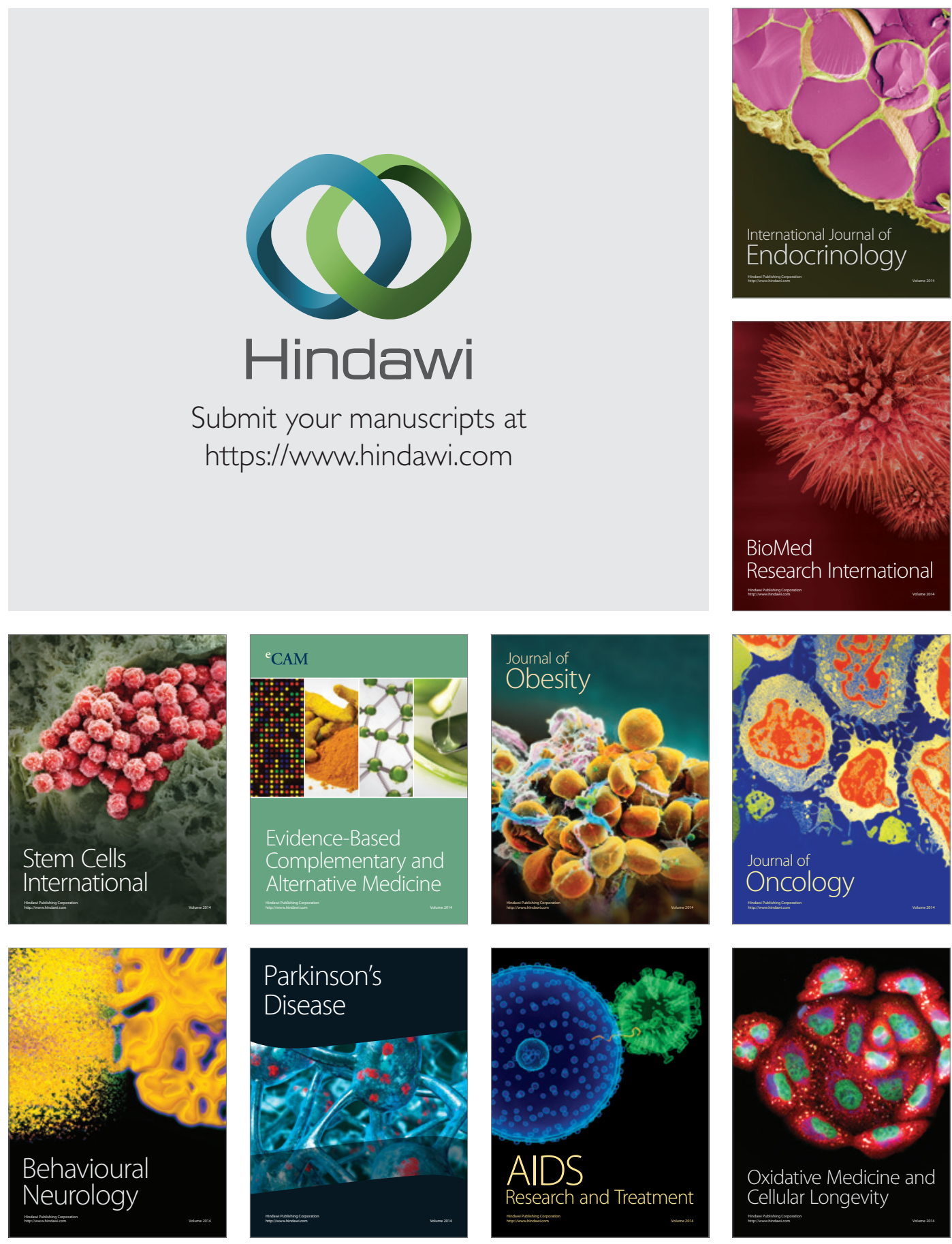